\title{
Expert consensus on the safety prevention and control of nuclear medicine diagnosis and treatment during the outbreak of COVID-19 (1st edition): (translated from Chinese version)
}

\author{
Xiaoli Lan $^{1} \cdot$ Yu Long ${ }^{1} \cdot$ Fuqiang Shao ${ }^{1} \cdot$ Yangminghui Song ${ }^{1}$ \\ Received: 19 May 2020 / Accepted: 1 June 2020 / Published online: 4 January 2021 \\ (C) The Author(s), under exclusive licence to Springer-Verlag GmbH Germany, part of Springer Nature 2021
}

\begin{abstract}
In December 2019, an infectious disease caused by a new type of coronavirus infection was prevalent in Wuhan and across the country. On January 20, 2020, the National Health Commission of the People's Republic of China issued No.1 Announcement, which incorporated the novel coronavirus pneumonia into the Class B infectious disease according to the Law on Prevention and Control of Infectious Disease, but the disease should be adopted in the management of Class A infectious disease. In order to effectively control the source of infection, cut off the transmission route, protect the susceptible population, ensure the medical quality and medical safety, perform epidemic prevention and control, and comprehensively guarantee the life safety and physical health of medical staff, patients, and family members, it is very important to organize and carry out nuclear medicine diagnosis and treatment scientifically and safely. According to the national prevention and control policy, Chinese Society of Nuclear Medicine and editorial board of the Chinese Journal of Nuclear Medicine and Molecular Imaging organized professionals to formulate the expert consensus on the safety prevention process of nuclear medicine imaging and nuclide therapy during the period of new coronavirus infection.
\end{abstract}

Keywords Coronavirus infections $\cdot$ Nuclear medicine $\cdot$ Protection and control

This article is part of the Topical Collection on Infection and inflammation The contributors of the original article are listed in the appendix

This article has been translated from Chinese. The original Chinese version has been published in the Chinese Journal of Nuclear Medicine and Molecular Imaging on February 24, 2020: Chin J Nucl Med Mol Imaging, 2020,40, DOI https://doi.org/10.3760/cma.j.cn321828-20200222-00062, URL http://rs.yiigle.com/yufabiao/1182642.htm (Translators (contributed equally, and sorted in alphabetical order by last name): Xiaoli Lan, Yu Long, Fuqiang Shao, Yangmeihui Song (Department of Nuclear Medicine, Union Hospital, Tongji Medical College, Huazhong University of Science and Technology)

Xiaoli Lan

xiaoli_lan@hust.edu.cn

Yu Long

longyu96_18@163.com

Fuqiang Shao

m15228213350@163.com

Yangminghui Song

songymh@hust.edu.cn

1 Department of Nuclear Medicine, Union Hospital, Tongji Medical College, Huazhong University of Science and Technology, Wuhan, China
Since December 2019, several hospitals in Wuhan, Hubei Province, China, reported an increasing number of cases of unexplained pneumonia, which were confirmed to be caused by a novel coronavirus, severe acute respiratory syndrome coronavirus 2 (SARS-CoV-2). The National Health Commission of the People's Republic of China and World Health Organization (WHO) officially named the disease as "novel coronavirus pneumonia", "Coronavirus Disease 2019" (COVID-19) [1]. The published data showed that COVID-19 is highly contagious $[2,3]$.

The practices in the Department of Nuclear Medicine, including nuclear medicine imaging and radionuclide therapy, are complex procedures, which involve various connections, different workplaces, and larger staffs. Therefore, the management of patients requires stringent criteria, and medical staff involved in the entire processes of diagnosis and treatment require strict protection. Basing on the characteristics of nuclear medicine imaging study and radionuclide therapy, the Chinese Society of Nuclear Medicine and the editorial board of the Chinese Journal of Nuclear Medicine and Molecular Imaging organized a group of professionals to formulate an Expert consensus on the safety prevention and control of nuclear medicine 
diagnosis and treatment during the outbreak of COVID-19 (1st edition) (from now on referred to as this consensus). This consensus is mainly based on the Diagnosis and Treatment Plan of Coronavirus Disease 2019 (Tentative Version 6) issued by the National Health Commission of PRC [4], the Technical Guidelines for the Prevention and Control of Novel Coronavirus (2019-ncov) Infection in Medical Institutions (First Edition) [5], the Guidelines for Usage of Common Medical Protective Products in Protection and Control of Novel Coronavirus Pneumonia (Tentative Edition) [6], the Protection Management and Procedures of Nuclear Medicine Imaging During Novel Coronavirus (2019-ncov) Infection Epidemic Period [7], and other relevant content. This consensus considers possible infection-related problems in the entire process of nuclear medicine practice and transmission routes of SARS-CoV-2. Moreover, it aims at instructing nuclear medicine medical staff to carry out effective safety prevention and control during the diagnosis and treatment, to minimize the infection of medical staff, to reduce the risk of virus transmission effectively, and to guarantee medical quality and safety during the COVID-19 epidemic. This consensus applies during the prevention and control of COVID-19 and when similar respiratory infectious diseases are encountered in the future.

\section{COVID-19 introduction}

\section{Pathogenic characteristics}

Coronavirus subfamilies are categorized into four genera: $\alpha$, $\beta, \gamma$ and $\delta$. The novel coronavirus SARS-CoV-2 belongs to the genus $\beta$ and is $96 \%$ identical to the genome of bat-SARSlike coronavirus [8, 9]. Most coronaviruses cause upper respiratory tract infections; SARS-CoV-2 can cause mild and even severe pneumonia and is highly contagious. Coronaviruses are sensitive to ultraviolet rays and heat. The viruses can be effectively inactivated by heating at $56{ }^{\circ} \mathrm{C}$ for $30 \mathrm{~min}$. Most disinfectants (including $75 \%$ ethanol and chlorine-containing disinfectant) can effectively inactivate viruses. However, chlorhexidine cannot effectively inactivate viruses and should not be used [2].

\section{Epidemiological characteristics}

The major sources of infection are patients with COVID-19, including asymptomatic carriers. COVID-19 is highly contagious, which is mainly transmitted by respiratory droplets and contact. Humans of all ages are generally susceptible. Other routes, including aerosol and digestive tract transmission, are yet to be clarified [4].

\section{Clinical characteristics}

The incubation period of COVID-19 is between 1 to 14 days, mostly within 3 to 7 days. The main clinical manifestations of the disease include fever, fatigue, and dry coughing. Symptoms such as stuffy nose, runny nose, pharyngitis, myalgia, and diarrhea are less common. Mild cases are only presenting with light fever and mild fatigue. In severe cases, dyspnea and/or hypoxemia usually occur after 1 week from the onset, and worse cases can rapidly progress to acute respiratory distress syndrome, septic shock, metabolic acidosis that is hard to correct, coagulation dysfunction, and others [4].

\section{Diagnostic criteria [4]}

Suspected cases The suspected cases should be diagnosed by considering epidemiological histories and clinical manifestations.

Confirmed cases Suspected cases with one of the following etiological pieces of evidence can be diagnosed as confirmed cases: (1) a positive result of the nucleic acid of SARS-CoV-2 by real-time fluorescence RT-PCR and (2) the virus gene sequence is high homologous to the known SARS-CoV-2.

\section{Work objectives and prevention and control principles}

While ensuring the quality of diagnosis and treatment during nuclear medicine practice, we aim to achieve "zero infection" among medical staff and to prevent cross-infection between patients. The general principles for the prevention and control of SARS-CoV-2 infection are as follows: control the source, cutting off transmission routes, standardize the prevention and control measures, and strengthening environmental disinfection.

\section{Control the source}

It is necessary to quickly identify potentially at-risk, suspected, and confirmed patients and carry out unique management of these patients, respectively.

\section{Cut off transmission routes}

Because droplets and close contact mainly transmit SARSCoV-2, both doctors and patients need to wear masks and strengthen hand hygiene. SARS-CoV-2 has the potential to spread through aerosol, digestive tract, and excreta. Therefore, it is necessary to disinfect the air, body fluids, and excreta in the controlled areas. 


\section{Standardize the prevention and control measures}

Refer to the Guidelines for Usage of Common Medical Protective Products in Protection and Control of Novel Coronavirus Pneumonia (Tentative Edition) to correctly use the medical protective products, to standardize prevention and control, and to avoid infection [6].

\section{Strengthen environmental disinfection}

The areas and instruments with potential pollution caused by the suspected or confirmed patients should be effectively sterilized to destroy the virus growth environment.

\section{Screening and classification of patients during nuclear medicine diagnosis and treatment procedures and suggestions for prevention and control of diagnosis and treatment}

\section{Screen and classify patients}

It is essential to verify patients' information and screen them precisely during the diagnostic and therapeutic procedures. Patients can be divided into three groups: ordinary patients, potential at-risk patients, and suspected or confirmed COVID-19 patients. This classification is done according to their travel history, contact history, clinical manifestations, imaging examination, and nucleic acid test [4] (Fig. 1). The suspected COVID-19 patients identified during the triage procedure should be reported to the hospital infection management department immediately.

\section{Suggestion for prevention and control}

\section{Nuclear medicine imaging}

When suspected or confirmed COVID-19 patients are identified during the triage and come for general nuclear medicine examination, it is recommended to postpone the examination. Patients should be persuaded to postpone the examination, focus on treating COVID-19, and reschedule until their disease is cured and the epidemic is controlled. If the nuclear medicine examination is related to an emergency or is needed for an essential diagnosis and treatment, a reasonable waiting time should be defined according to the therapeutic strategy. Examinations of COVID-19 patients should be scheduled as the last one of the day. The number of patients and the interval between examinations should be monitored during that day.

During the epidemic period, it is recommended that the sequence of nuclear medical imaging examination for the patients in the above different groups should be ordinary patients, potential at-risk patients, and finally suspected or confirmed COVID-19 patients. During the examination, try to ensure that there is no contact between the patients in each group and all patients have to maintain an appropriate distance from each other, to avoid cross-infection.

\section{Radionuclide therapy}

Ordinary patients Radionuclide therapy can be given as scheduled, but patients must pay attention to personal protection and wear masks while undergoing any examination and treatment during hospitalization.

Potential at-risk patients Radionuclide therapy can be performed. Patients should stay in an isolated room and should not be allowed to enter public areas of the treatment ward. During the treatment, medical staff should strictly check for symptoms: fever, cough, diarrhea, and others. If symptoms appear, the ward should be immediately arranged following standards of the isolation ward. The patients should be studied with blood routine examination, lung CT, nucleic acid test, and others. The infectious disease department should be called promptly.

Confirmed or suspected COVID-19 patients It is recommended to postpone the radionuclide therapy. Patients should be instructed to resume routine medication and transferred to isolation wards or designated hospitals with effective isolation and treatment capacity to manage COVID-19 patients.

During the epidemic period, it is recommended that the patients in the different groups should be treated in an isolated room to minimize possible cross-infection in the hospital.

\section{In vitro analysis}

In China, the Biosafety Consensus for 2019 Novel Coronavirus Pneumonia in Clinical Laboratory [10] formulated by the Chinese Society of Laboratory Medicine Branch of the Chinese Medical Association is available for reference.

\section{Protection requirements for medical staff during nuclear medicine diagnosis and treatment process}

\section{Protection level and requirements of medical staff}

In addition to conventional radiation protection, for different groups of patients, all medical staff in contact with patients should adopt different protection requirements according to the protection level. It should be noted that the second level of protection is recommended for medical staff and associated personnel involved with patient transfer and garbage disposal for confirmed/suspected COVID-19 cases. The specific 
Fig. 1 Recognize and classify patients during nuclear medicine practice

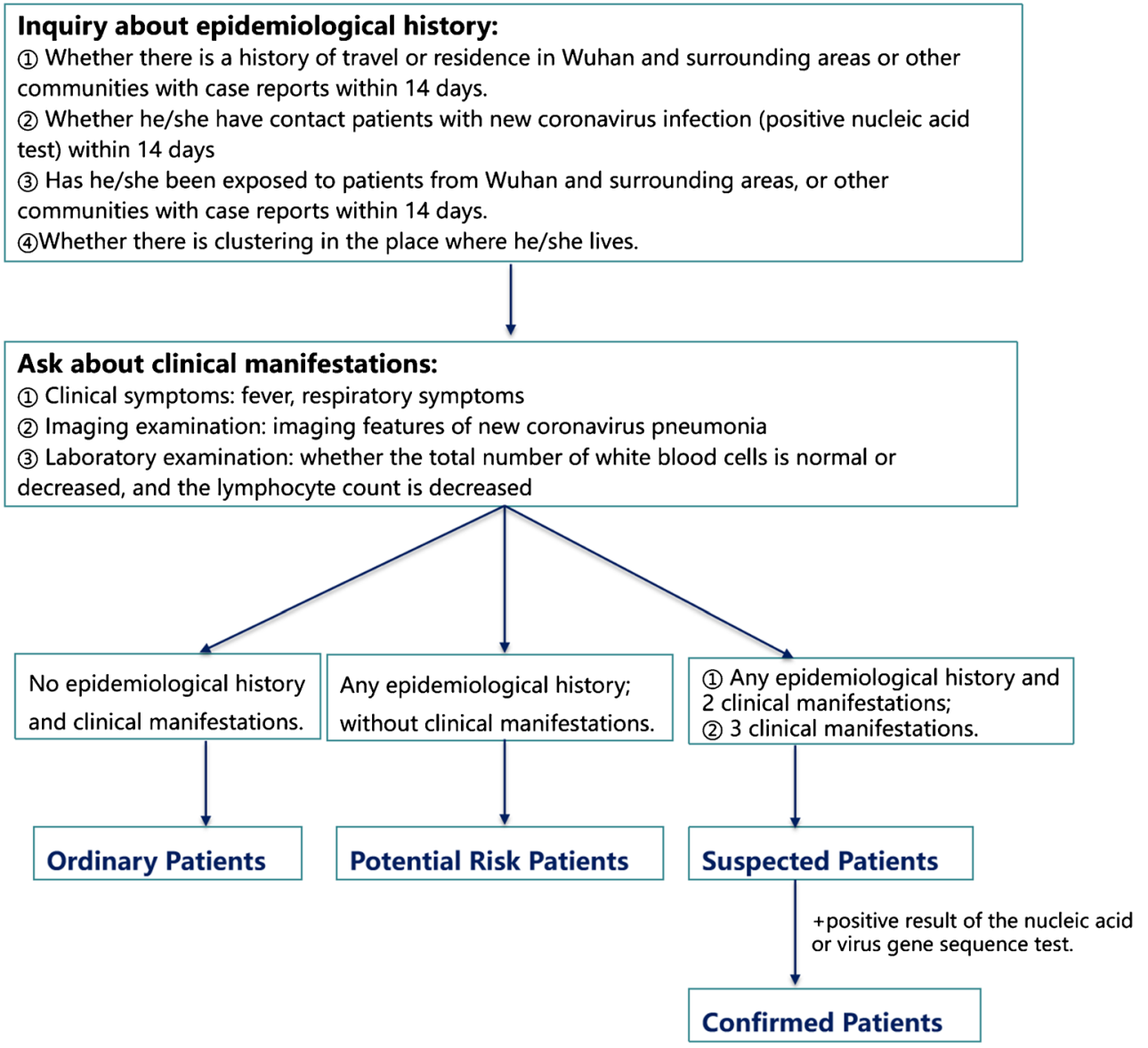

Inquiry about epidemiological history:

(1) Whether there is a history of travel or residence in Wuhan and surrounding areas or other communities with case reports within 14 days.

test) within 14 days

(3) Has he/she been exposed to patients from Wuhan and surrounding areas, or othe communities with case reports within 14 days.

(4) Whether there is clustering in the place where he/she lives. content of the protection requirements for medical staff is shown in Table 1.

\section{Protection precautions}

The key points of personal protection for medical staff includes wearing masks correctly, performing proper hand hygiene, and standardized personal protection

(1) During the diagnosis and treatment activities, all medical staff must wear medical gown, caps, and surgical masks correctly. For methods of wearing masks, refer to the Technical guidelines for the selection and use of masks to prevent novel coronavirus infection in different populations [11] formulated by the National Health Commission.

(2) After each contact with the patient, the medical staff must immediately perform hand hygiene or disinfection. Refer to the Specification of Hand Hygiene for Healthcare Workers [12]. Follow the six-step hand-washing method strictly and use quick-drying hand disinfectant (bromogeramine, 75\% alcohol, compound disinfectant, and others) to rub hands for 1 3 $\mathrm{min}$.
Table 1 Protection measurements for medical staff

Protection Patient classification Protective equipment level

\begin{tabular}{|c|c|c|}
\hline Level 3 & $\begin{array}{l}\text { Severe confirmed } \\
\text { COVID-19 patients } \\
\text { Suspected and confirmed } \\
\text { COVID-19 patients who } \\
\text { need to receive injection, } \\
\text { sputum suctioning, and } \\
\text { specimen collecting } \\
\text { (blood drawing, percuta- } \\
\text { neous biopsy) }\end{array}$ & $\begin{array}{l}\text { Disposable medical cap, } \\
\text { protective glasses or face } \\
\text { shield (antifog type), } \\
\text { medical protective mask } \\
\text { (N95), isolation gown, } \\
\text { disposable latex gloves, } \\
\text { disposable shoe covers, } \\
\text { and protective face mask } \\
\text { (or full-face air respirator, } \\
\text { or positive pressure head- } \\
\text { gear) }\end{array}$ \\
\hline Level 2 & $\begin{array}{l}\text { Patients with potential risk } \\
\text { Suspected and confirmed } \\
\text { patients }\end{array}$ & $\begin{array}{l}\text { Disposable medical cap, } \\
\text { protective glasses or face } \\
\text { shield (antifog type), } \\
\text { medical protective mask } \\
\text { (N95), isolation gown, } \\
\text { disposable latex gloves, } \\
\text { disposable shoe covers }\end{array}$ \\
\hline Level 1 & Ordinary patients & $\begin{array}{l}\text { Medical gown, caps, and } \\
\text { surgical masks }\end{array}$ \\
\hline
\end{tabular}


(3) For the second and third level protection, strictly follow the procedures for wearing and taking off personal protective equipment, which is implemented following the Technical Guidelines for the Prevention and Control of Novel Coronavirus Infection (2019-ncov) in Medical Institutions (First Edition) [5].

\section{Avoiding cross-infection}

(1) All medical staff cannot remove the mask in the contaminated area or bring the medical gown and caps used in contaminated area to the clean area.

(2) It is prohibited to wear personal protective equipment while leaving the contaminated area, to avoid crossinfection in various areas.

(3) After contact with suspected or confirmed COVID-19 patients and their belongings, medical staff must not touch keyboards of imaging instruments and other clean facilities, before disinfection, to prevent the expansion of medical pollution.

(4) It is recommended that medical staff and suspected or confirmed COVID-19 patients follow the dual-channel process in diagnosis and treatment.

(5) The medical staff should work in their workplace and abstain from walking around and talking with each other.

\section{Protection requirements for patients during nuclear medicine diagnosis and treatment process}

(1) Patients and accompanying persons must not hide medical history and need to provide information such as epidemiological history, clinical symptoms, image diagnosis, laboratory examination, and nucleic acid or gene test.

(2) During the imaging examination, patients should not be accompanied when their conditions allow. If necessary, each patient is limited to one accompanying person, and children or patients with difficulties are limited up to two.

(3) Patients and accompanying persons must wear masks throughout the consultation and follow the medical staff's instructions.

(4) Both patients and accompanying persons are required to monitor the body temperature.

(5) All patients must stay no less than $1 \mathrm{~m}$ from each other while waiting.

(6) The suspected or confirmed COVID-19 patients who have to undergo nuclear medicine examinations are placed in an isolated room for waiting, to prevent patients from walking around during the entire diagnosis and treatment process.
(7) Instruct patients and accompanying persons not to spit anywhere and put water cups and domestic waste into designated garbage cans.

(8) Inpatients for radionuclide therapy must stay in isolated rooms alone and are strictly prohibited from visiting each other, staying or gathering in the public areas of the ward, or going out during hospitalization. Visits and relatives from outside the hospital are also strictly prohibited.

\section{Protection and control in workflow}

The workflow of nuclear medicine imaging (Fig. 2, upper row) involves many procedures and takes a long time. Doctors, technicians, and nurses are all in possible in contact with patients. The workflow of radionuclide therapy flowchart (Fig. 2, lower row) has a similar process to nuclear medicine imaging (such as appointment, admission, imaging tracer injection or therapeutic radionuclide administration, imaging acquisition), which also needs special protection management during COVID-19 epidemic period.

The relevant sites involved in nuclear medicine imaging are shown in Fig. 3. Contaminated areas and clean areas should be clearly divided. All items in contaminated areas should not be brought into clean areas without disinfection.

\section{Appointment}

(1) Screen ordinary patients, potential at-risk patients, and suspected or confirmed COVID-19 patients, and schedule examination or treatment time according to different patient types.

(2) Control the number of patients and the interval between examinations.

(3) Accompanying the patient is not recommended when the patient condition permits. If necessary, each patient is limited to one accompanying relative, and children or people with limited mobility are up to two.

(4) Patients and accompanying persons should wear medical protective masks.

\section{Admission and inquiry}

(1) Further identification and classification of the patient are required.

(2) The body temperature of patients and accompanying persons should be checked.

(3) Admission or information personnel should keep an appropriate distance (no less than $1 \mathrm{~m}$ ) from patients and see one patient or their relative at a time. 
Fig. 2 The workflow of radiopharmaceutical imaging (upper row) and radionuclide therapy (lower row)

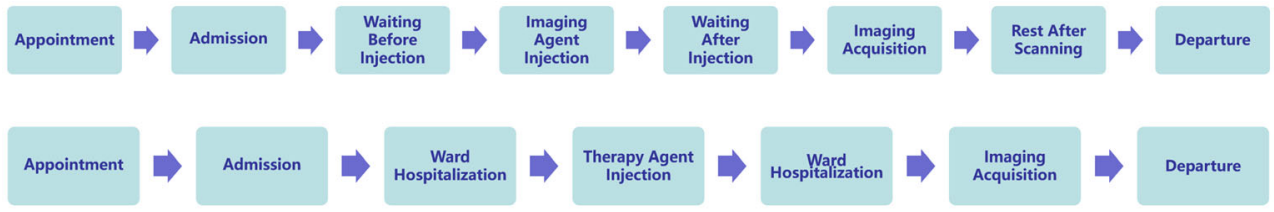

(4) Separate waiting rooms for suspected or confirmed patients should be available. Patients are required to stay in the designated area throughout the examination and not to walk around, to avoid contacts with other patients.

(5) Strengthen doctor-patient communication and patients' psychological counseling to eliminate patients' anxiety and fear, thereby ensuring the smooth implementation of the diagnosis and treatment.

(6) Disinfect the work environment and work surfaces timely after receiving patients.

\section{Imaging tracer injection (therapeutic radiopharmaceuticals administration)}

(1) One pad towel and one tourniquet for one person for injections or one cup for one person for oral administration.

(2) Hand disinfection should be performed after the injection or administration of the previous patient and before the next patient.
(3) Used infusion sets, syringe, cotton swab, and dressing should be placed into dedicated containers for centralized destruction.

(4) Disinfect the surface of glucometer timely after measuring a patient's blood glucose before the PET examination.

(5) At the end of the operation, the surface of the operation table and used equipment and the air in the injection room and waiting room should be disinfected.

\section{Operation during imaging examination}

(1) Patients should wear disposable shoe covers before image acquisition. Put a disposable sheet on the examining table.

(2) Replace the disposable sheet on the examining table after each examination. Disinfect the areas the patient has touched, such as examining table and the surface coil of PET/MR (see disinfection methods in section seven).

Fig. 3 The procedures and workplaces in nuclear medicine imaging study

\section{Potentially contaminated area \\ Patient Only Interaction}

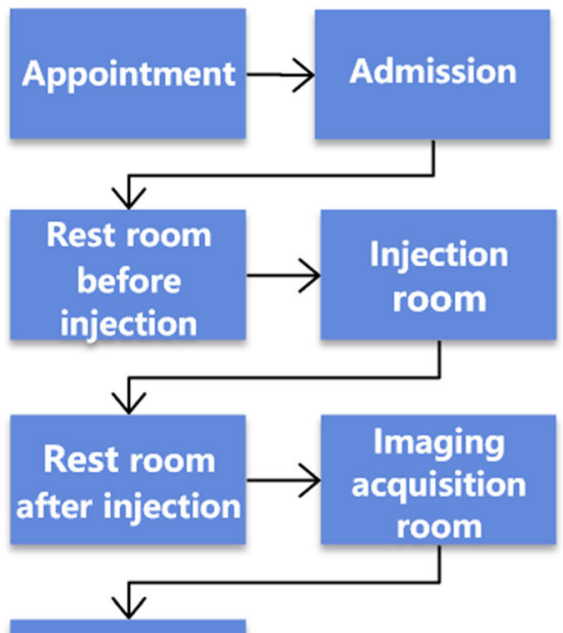

Rest room

after scanning

$\downarrow$

Departure
Clean area

Medics Only

Imaging

diagnosis room

Office 
(3) Technicians should urge and confirm the patient to wear a mask throughout the examination to prevent droplets from entering the instrument detector and the machine room air. Pay attention to whether there is a metal wire in the mask if a PET/MR scan is to be performed, especially for the head and neck PET/MR scan. If so, remove the metal wire from the mask or replace a wireless mask.

(4) During the scanning process, if the technicians find that their protective equipment (such as masks, goggles, gloves, and protective clothing) are contaminated (by blood, fluids, secretions), they should replace this equipment in time and wash and sanitize their hands.

\section{Special management of patients receiving radionuclide therapy during hospitalization}

(1) Strictly screen inpatients, and prevent the admission and treatment of suspected and confirmed COVID-19 patients. Suspected and confirmed COVID-19 patients should be instructed to resume routine treatment and transfer to designated hospitals or isolation wards with effective isolation and treatment capacity for the diagnosis and treatment of COVID-19.

(2) All inpatients (or legal custodians) must truthfully fill in Letter of commitment for Novel Coronavirus Infection Notification.

(3) Temperature monitoring: One thermometer for one person. Measure and record the body temperature of patients regularly every day.

(4) Observe patients for symptoms like unexplained fever, cough, and diarrhea during the treatment. Once the symptoms mentioned above occur, treat immediately following standards and methods of the isolation ward; perform blood routine examination, lung CT, nucleic acid test, and other related examinations for the patients; and request the infectious disease department or respiratory department for in-hospital consultation.

(5) Strengthen health education for patients, especially the education of COVID-19-related self-protection knowledge.

(6) Hospital distributes meals and medicines for patients uniformly. Medical staff arrange the order of taking food and medicines, only one person at a time.

(7) It is recommended to strengthen the application of electronic information management of patients and provide notification instructions and information to patients, to minimize the direct contact between medical staff and patients.

(8) When suspected or confirmed COVID-19 patients are transferred to other department or hospital, the hospital infection office should assign professionals to conduct terminal disinfection of the environment referring to the Technical Standards for Disinfection in Medical Institutions, in Technical Guidelines for Prevention and Control of Novel Coronavirus (2019-ncov) Infection in Medical Institutions (First Edition) [5].

\section{Protection and control after workflow}

Relevant suggestions were mainly based on the Technical Guidelines for the Prevention and Control of Novel Coronavirus Infection (2019-ncov) in Medical Institutions (First Edition) [5] and the Regulation for Cleaning and Disinfection Management of Environmental Surface in Healthcare [13] and combined with the characteristics of nuclear medical instruments. The cleaning equipment in contaminated areas and clean areas should be strictly distinguished, and mixing use is prohibited.

\section{Imaging instrument disinfection}

Instrument disinfection should be performed to prevent SARS-CoV-2 from adhering to the surface of the instruments or being in the scanning room air. According to the Diagnosis and Treatment Plan of Coronavirus Disease 2019 (draft of the sixth revised edition) [4], it is recommended to dip the soft cloth in $75 \%$ of medical alcohol to wipe the surface of instruments and let it dry naturally. The cleaning procedure should be applied to the surface exposed to the air, especially the equipment surface in direct contact with the patient, such as examining table, breathing band, detector surface, and PET/ MR coil. Electronic circuit interface like PET/MR surface coil interface is also exposed to air. As there is electronic circuit pin or pinhole, it is recommended to wipe and disinfect with anhydrous ethanol or equipment recommended products and let it dry naturally. Sprays should not be used to disinfect medical instruments, to prevent the disinfectant from penetrating the instrument and causing short circuit and corrosion.

\section{Imaging examination environment disinfection}

(1) If possible, place a mobile air disinfecting machine in the machine room to kill coronavirus in air. However, due to the existence of a strong magnetic field in PET/MR machine room, placing the air disinfecting machine is forbidden. The air disinfecting machine could be placed at the entrance of the air conditioning in the equipment room because the air between PET/MR machine room and equipment room recirculates internally. Maintain the air humidity within the required humidity range, generally no higher than $60 \%$. 
(2) The floor of the scanning room and operation room should also be disinfected, and $1000 \mathrm{mg} / \mathrm{L}$ chlorinecontaining disinfectant is recommended. Visible pollutants should be completely removed with disposable absorbent materials before disinfection. Disinfect at any time in case of contamination.

\section{Terminal disinfection of radionuclide treatment ward}

\section{Cleaning and disinfection of floor and objects' surfaces}

(1) When there is no visible contamination, conduct wet cleaning.

(2) When the floor and objects' surfaces are contaminated by the patient's blood and body fluids, remove the visible contamination with disposable absorbent materials and then disinfect with $2000 \mathrm{mg} / \mathrm{L}$ chlorine-containing disinfectant for $30 \mathrm{~min}$.

\section{Cleaning and disinfection of indoor air}

(1) Close the doors and windows before disinfection. Use hydrogen peroxide air sterilizer with $3 \%$ hydrogen peroxide solution for spraying disinfection; a spray volume of $20 \sim 30 \mathrm{ml} / \mathrm{m}^{3}$ is recommended.

(2) Disinfect for 30 60 min, and then open the doors and windows for thorough ventilation.

\section{Matters that need attention}

(1) Disinfection personnel should also arrange individual protection.

(2) Divide clean areas and contaminated areas according to the contamination situation.

(3) During terminal disinfection, the passage leading to the area to be sterilized should be disinfected first.

(4) Indoor disinfection should be completed from the outside to inside and up to down. Moreover, clean the area with severe contamination first, and then begin disinfection to door, ground, furniture, and wall.

(5) For respiratory infectious disease, focus on air disinfection (close doors and windows for airtight disinfection) and ventilate timely after disinfection.

\section{Disinfection in other potentially contaminated areas}

(1) Disinfect the toilets with prepared 84 disinfectants twice a day.
(2) Disinfect the handrails, faucets, and other areas possibly touched by patients with prepared 84 disinfectants.

\section{Waste management}

(1) All waste from patients should be disposed of as infectious medical waste, strictly complying with the Regulations for the Medical Waste Management and the Measures for the Administration of Medical Waste in Medical and Health Institutions $[14,15]$. When infectious medical waste is simultaneously radioactive, refer to the Regulations on the Safety Management of Radioactive Waste [16].

(2) After inspection, the protective equipment should be put directly into the clinical waste bucket, requiring doublelayer sealing, clear marking, and airtight transportation.

\section{Compliance with ethical standards}

Conflict of interest The authors declare that they have no conflict of interest.

Research involving human participants and/or animals No human participants and animals were involved in this research.

Informed consent No informed consent needed in this guideline.

\section{Appendix}

\section{Chinese Society of Nuclear Medicine to Chinese Society of Nuclear Medicine, Editorial Board of Chinese Journal of Nuclear Medicine and Molecular Imaging}

\section{Experts in writing:}

Xiaoli Lan, M.D, Ph.D (Department of Nuclear Medicine, Union Hospital, Tongji Medical College, Huazhong University of Science and Technology; xiaoli_lan@hust.edu.cn)

Zairong Gao, M.D, Ph.D (Department of Nuclear Medicine, Union Hospital, Tongji Medical College, Huazhong University of Science and Technology; gaobonn@163.com)

Zhi Yang, Ph.D (Department of Nuclear Medicine, Key Laboratory of Malignant Tumor Pathogenesis and Translational Research, Ministry of Education, Peking University Cancer Hospital \& Institute; pekyz@ 163.com)

Yamin Li (Department of Nuclear Medicine, the First Hospital Affiliated to China Medical University; ymli2001@163.com)

Sijin Li (Department of Nuclear Medicine, First Hospital of Shanxi Medical University; lisjnm123@163.com) 
Corresponding authors: Sijin Li M.D, Ph.D (lisjnm123@163.com)

Yaming Li, M.D, Ph.D (ymli2001@163.com)

\section{Reviewers (in alphabetical order by last name):}

Rui An (Department of Nuclear Medicine, Union Hospital, Tongji Medical College, Huazhong University of Science and Technology; anruiwh@163.com)

Hong Ding (Editorial Department, Chinese Journal of Nuclear Medicine and Molecular Imaging; 13906188451@ 139.com)

Huang Gang (Shanghai Key Laboratory of Molecular Imaging, Shanghai University of Medicine and Health Science; huang2802@163.com)

Lin Li (Department of Nuclear Medicine, West China Hospital, Sichuan University; lilinhuaxi@sina.com)

Sijin Li (Department of Nuclear Medicine, First Hospital of Shanxi Medical University; lisjnm123@163.com)

Yamin Li (Department of Nuclear Medicine, the First Hospital Affiliated to China Medical University; ymli2001@ 163.com)

Hongcheng Shi (Department of Nuclear Medicine, Zhongshan Hospital, Fudan University; shihongcheng@ sina.com)

Jing Wang (Department of Nuclear Medicine, Xijing Hospital,

Fourth Military Medical University; wangjing@ fmmu.edu.cn)

Hui Wang (Department of Nuclear Medicine, Xinhua Hospital, Shanghai Jiaotong University School of Medicine; wanghuishanghai@hotmail.com )

Baixuan Xu (Department of Nuclear Medicine, General Hospital of the PLA; xbx301@163.com)

Yongxue Zhang (Department of Nuclear Medicine, Union Medical College Hospital, Tongji Medical College, Huazhong University of Science and Technology; zhyx1229@163.com)

\section{Compilation expert group members (sorted in alphabetical order by last name):}

Rui An (Department of Nuclear Medicine, Union Hospital, Tongji Medical College, Huazhong University of Science and Technology; anruiwh@163.com)

Haidong Cai (Department of Nuclear Medicine, Shanghai Tenth People's Hospital; caihdong@163.com)

Zairong Gao (Department of Nuclear Medicine, Union Hospital, Tongji Medical College, Huazhong University of Science and Technology; gaobonn@163.com)

Zhongke Huang (Department of Nuclear Medicine, Sir run run Shaw Hospital, Zhejiang University School of Medicine; 513893839@qq.com)

Li Huo (Department of Nuclear Medicine, Peking Union Medical College Hospital, Chinese Academy of Medical Sciences, Beijing Key Laboratory of Molecular Targeted Diagnosis and Therapy in Nuclear Medicine; huoli@pumch.cn)
Xiaoli Lan (Department of Nuclear Medicine, Union Hospital, Tongji Medical College, Huazhong University of Science and Technology; xiaoli_lan@ hust.edu.cn)

Xiao Lei (Department of Nuclear Medicine, Youanmen Hospital, Fengtai, Beijing; leixiao@aliyun.com)

Cen Lou (Department of Nuclear Medicine, Sir run run Shaw Hospital, Zhejiang University School of Medicine; 3194110@zju.edu.cn)

Keyi Lu (Department of Nuclear Medicine, First Hospital of Shanxi Medical University; lu-ky@163.com)

Zhongwei Lv (Department of Nuclear Medicine, Shanghai No.10 People's Hospital; shtjnmd@163.com)

Yunchuan Ma (Department of Nuclear Medicine, Xuanwu Hospital, Capital Medical University; mayc_home@163.com)

$\mathrm{Na}$ Niu (Department of Nuclear Medicine, Peking Union Medical College Hospital, Chinese Academy of Medical Sciences, Beijing Key Laboratory of Molecular Targeted Diagnosis and Therapy in Nuclear Medicine; niunayy@sina.com)

Hongcheng Shi (Department of Nuclear Medicine, Zhongshan Hospital, Fudan University; shihongcheng@ sina.com)

Chunmei Wang (Department of Nuclear Medicine, Affiliated Hospital of Inner Mongolia Medical University; wangchunmei@163.com)

Feng Wang (Department of Nuclear Medicine, Key Laboratory of Malignant Tumor Pathogenesis and Translational Research, Ministry of Education, Peking University Cancer Hospital \& Institute; windtinerwf@ 163.com)

Xuemei Wang (Department of Nuclear Medicine, Affiliated Hospital of Inner Mongolia Medical University; wangxuemei201010@163.com)

Yan Xiu (Department of Nuclear Medicine, Zhongshan Hospital, Fudan University; xiuyan1112@vip.sina.com)

Zhi Yang (Department of Nuclear Medicine, Peking University Cancer Hospital and Beijing Cancer Institute, Key Laboratory of Malignant Tumor Pathogenesis and Translational Research, Ministry of Education; pekyz@163.com)

Yongxue Zhang (Department of Nuclear Medicine, Union Hospital, Tongji Medical College, Huazhong University of Science and Technology; zhyx1229@163)

\section{References}

1. Coronavirus disease officially named COVID-19 [DB / OL]. (2020-02-11) [2020-02-21] https://www.nature.com/articles/ d41586-020-00154-w. Accessed 29 Jun 2020.

2. World Health Organization. Clinical management of severe acute respiratory infection when novel coronavirus (2019-nCoV) infection is suspected: interim guidance, 28 January 2020 [DB/OL] (2020-01-28) [2020-02-21] https://apps.who.int/iris/handle/10665/ 330893. Accessed 29 Jun 2020.

3. Huang CL, Wang YM, Li XW, et al. Clinical features of patients infected with 2019 novel coronavirus in Wuhan, China. Lancet. 
2020;395(10223):497-506. https://doi.org/10.1016/S01406736(20)30183-5.

4. National Health Committee of the People's Republic of China. Diagnosis and treatment plan of coronavirus disease 2019 (Tentative Version 6) [EB / OL]. (2020-02-18) [2020-02-21]. http://www.nhc. gov.cn/xcs/zhengcwj/202002/8334a8326dd94d329df351d7da8aefc2/ files/b218cfeb1bc54639af227f922bf6b817.pdf. Accessed 29 Jun 2020.

5. National Health Committee of the People's Republic of China. Technical guidelines for the prevention and control of novel coronavirus (2019-ncov) infection in medical institutions (First Edition) [EB / OL]. (2020-01-22) [2020-02-21] http:// www.gov.cn/ zhengce/zhengceku/2020-01/23/content_5471857.htm. Accessed 29 Jun 2020.

6. The National Health Committee of People's Republic of China. Guidelines for usage of common medical protective products in protection and control of novel coronavirus pneumonia (tentative edition) [EB / OL] (2020-01-26) [2020-02-21] http://www.nhc.gov. $\mathrm{cn} / \mathrm{xcs} /$ zhengcwj/202001/e71c5de925a64eafbe1 ce790debab5c6. shtml. Accessed 29 Jun 2020.

7. Lan XL, Sun X, Qin CX, et al. Protection management and procedures of nuclear medicine imaging during novel coronavirus (2019nCov) infection epidemic period. Chin J Nucl Med Mol Imaging. 2020;40(2):105-7. https://doi.org/10.3760/cma.j.issn.2095-2848. 2020.02.010.

8. Zhou P, Yang XL, Wang XG, et al. A pneumonia outbreak associated with a new coronavirus of probable bat origin. Nature, 2020. (2020-02-03) [2020-02-21]. https://doi.org/10.1038/s41586-0202012-7 https://www.nature.com/articles/s41586-020-2012-7. Accessed 29 Jun 2020.

9. Wu F, Zhao S, Yu B, et al. A new coronavirus associated with human respiratory disease in China. Nature, 2020. (2020-02-03) [2020-02-21]. https://doi.org/10.1038/s41586-020-2008-3 https:// www.nature.com/articles/s41586-020-2008-3. Accessed 29 Jun 2020.

10. Chinese Society of Laboratory Medicine. Biosafety consensus for 2019 novel coronavirus pneumonia in clinical laboratory. Chin J Lab Med. 2020;43(00):E001-E001 [2020-02-21]. https://doi.org/ 10.3760/cma.j.issn.1009-8158.2020.0001 http://rs.yiigle.com/ yufabiao/1180119.htm. Accessed 29 Jun 2020.

11. The State Council's joint prevention and control mechanism for pneumonia epidemic in response to new coronavirus infection. Technical guidelines for the selection and use of masks to prevent novel coronavirus infection in different populations [EB/OL]. (2020-02-04) [2020-02-21] http: //www.nhc.gov.cn/jkj/s7916/ 202002/485e5bd019924087a5614c4f1db135a2.shtml. Accessed 29 Jun 2020.

12. National Health Committee of the People's Republic of China. WS/ T 311-2019 Specification of hand hygiene for healthcare workers [S / OL]. (2019-11-26) [2020-02-21]. http://www.nhc.gov.cn/wjw/ s9496/202002/dbd143c44abd4de8b59a235feef7d75e/files/ 6a3e2bf3d82b4ee8a718dbfc3cde8338.pdf. Accessed 29 Jun 2020.

13. National Health and Family Planning Commission of the People's Republic of China. WS/T 512-2016 Regulation for cleaning and disinfection management of environmental surface in healthcare [S/OL]. (2016-12-27) [2020-02-21] http://www.nhc.gov.cn/ ewebeditor/uploadfile/2017/01/20170119150706183.pdf. Accessed 29 Jun 2020.

14. State Council of the People's Republic of China. Regulations for the medical waste management [Z/OL]. (2011-01-08) [2020-0221] http://www.gov.cn/gongbao/content/2011/content_1860802.htm. Accessed 29 Jun 2020.

15. Ministry of Health of the People's Republic of China. Measures for the administration of medical waste in medical and health institutions [DB/OL]. (2003-10-15) [2020-02-21] http://www.nhc.gov. cn/fzs/s3576/ 201808 / fb4c9e59b0cf45c3843ad585b30b0c6d. shtml. Accessed 29 Jun 2020.

16. State Council People's Republic of China. Regulations on the safety management of radioactive waste [the Z-OL]. (2011-12-20) [2020-02-21] http://www.gov.cn/zwgk/2011-12/29/content 2033177.htm. Accessed 29 Jun 2020.

Publisher's note Springer Nature remains neutral with regard to jurisdictional claims in published maps and institutional affiliations. 